\title{
Convex Polytopes, Algebraic Geometry, and Combinatorics
}
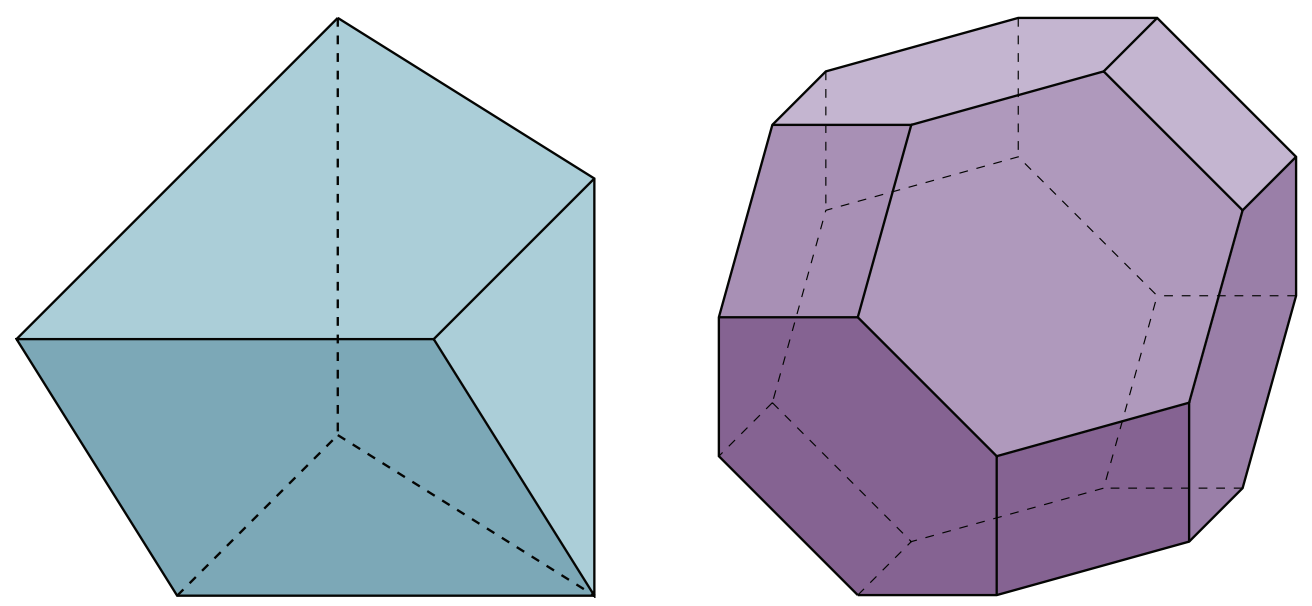

\section{Laura Escobar and Kiumars Kaveh}

In the last several decades, convex geometry methods have proven very useful in algebraic geometry specifically to understand discrete invariants of algebraic varieties. An approach to study algebraic varieties is to assign to a family of varieties a corresponding family of combinatorial objects which encode geometric information about the varieties. Often, the combinatorial objects that arise are convex polytopes, and convex geometry has been an essential tool for this strategy.

The emergence of convexity in algebraic geometry is rooted in the following geometric observations. Let $\mathcal{A} \subset$ $\mathbb{Z}^{n}$ be a finite set, then:

1. For any vector $\xi \in \mathbb{R}^{n}$, the maximum/minimum of the dot products $\xi \cdot x, x \in \mathcal{A}$, is attained on the boundary of the convex hull of $\mathcal{A}$.

2. As $k \rightarrow \infty$ the rescaled $k$-fold sums $\left\{\frac{1}{k}\left(x_{1}+\cdots+x_{k}\right) \mid\right.$ $\left.x_{i} \in \mathcal{A}\right\}$ converge to the convex hull of $\mathcal{A}$.

Laura Escobar is an assistant professor of mathematics at Washington University in St. Louis. Her email address is 1 aurae@wust 1 . edu. She was supported in part by NSF grant DMS-1855598.

Kiumars Kaveh is an associate professor of mathematics at the University of Pittsburgh. His email address is kaveh@pitt. edu. He was partially supported by NSF grant DMS-1601303, a Simons Foundation Collaboration Grant for Mathematicians, and a Simons Fellowship.

Communicated by Notices Associate Editor Stephan Ramon Garcia.

For permission to reprint this article, please contact:

reprint-permission@ams.org.

DOI: https://doi.org/10.1090/noti2137
The first observation is related to describing a polytope as an intersection of finitely many half spaces. It appears in the notion of a Newton polygon introduced in Section 1 and is important in tropical geometry. The second observation is related to the construction of a polytope as a convex hull of finitely many points. It is central to the proof of the BKK theorem (see Theorem 1.2) and its generalization to Newton-Okounkov bodies described in Section 3.

\section{Newton Polytopes and Toric Varieties}

The origin of appearances of convex polyhedra in algebraic geometry goes back to Sir Isaac Newton and Ferdinand Minding. Newton introduced what we now know as the Newton diagram of a polynomial $f(x, t)$ in two variables $x$ and $t$. Given an equation $f(x, t)=0$ regarded as implicitly defining $x$ in terms of $t$, Newton was interested in expressing $x$ as an infinite series in $t$. He knew that in general $x=x(t)$ may not be a power series but a series of the form $\sum_{i \geq 0} c_{i} t^{i / k}$ for some fixed integer $k>0$, a series with fractional exponents. In general one expects to have as many solutions as the degree of $f$ in $x$.

Let $f(x, t)=\sum_{a, b} c_{a, b} x^{a} t^{b}$. Consider the Newton diagram (or Newton polygon) of $f(x, t)$ to be the lower convex hull of $\left\{(a, b) \mid c_{a, b} \neq 0\right\} \subset \mathbb{R}^{2}$. This is the lower part of the boundary of the convex hull stretching from the leftmost to the rightmost point. Suppose the slopes of the (nonvertical) line segments in the Newton diagram are $\mu_{1}, \ldots, \mu_{s}$. Newton showed that the fractional exponents for the first terms in the series representations of solutions for $x$ are 


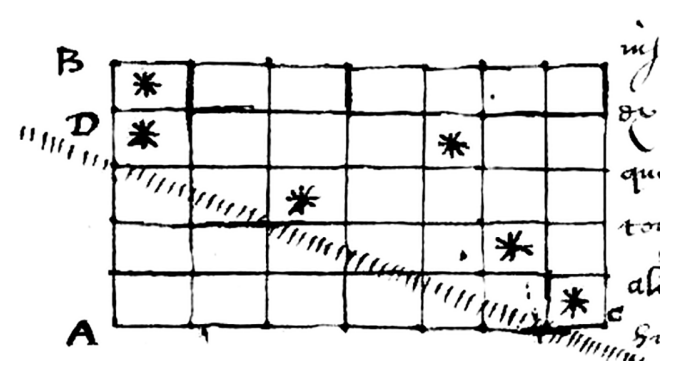

Figure 1. Example from Newton's letter to Oldenburg dated October 24, 1676.

the $-\mu_{i}$. This observation is a key step in proving the wellknown Newton-Puiseux Theorem, which describes the algebraic closure of the field of formal power series $\mathbb{C}((t))$ (see [MS15, Theorem 2.1.5]).

Motivated by Newton's classic work, Vladimir Arnold asked his students to work on generalizations and analogues of the notion of Newton diagram/polygon in several variables. This resulted in the modern definition of the Newton polytope of a polynomial. Unlike the Newton diagram, which is a local concept related to multiplicities of roots of a polynomial, the Newton polytope (Definition 1.1) is a global concept which can be thought of as a refinement of the notion of degree of a polynomial.

Throughout we denote the multiplicative group of nonzero complex numbers by $\mathbb{C}^{*}$. The product $\left(\mathbb{C}^{*}\right)^{n}$ of $n$ copies of $\mathbb{C}^{*}$ is an algebraic group often called an algebraic torus (it contains the usual topological torus $\left(S^{1}\right)^{n}$ as a maximal compact subgroup). The algebra of regular functions on $\left(\mathbb{C}^{*}\right)^{n}$ is the algebra of Laurent polynomials $\mathbb{C}\left[x_{1}^{ \pm}, \ldots, x_{n}^{ \pm}\right]$. We will use the multi-index notation, and for $\alpha=\left(a_{1}, \ldots, a_{n}\right) \in \mathbb{Z}^{n}$ we write $x^{\alpha}$ to denote the monomial $x_{1}^{a_{1}} \cdots x_{n}^{a_{n}}$.

Definition 1.1. Let $f(x)=\sum_{\alpha} c_{\alpha} x^{\alpha}$ be a Laurent polynomial. The Newton polytope of $f$ is the convex hull of the finite set $\left\{\alpha \mid c_{\alpha} \neq 0\right\}$. It is a convex polytope with vertices in $\mathbb{Z}^{n}$.

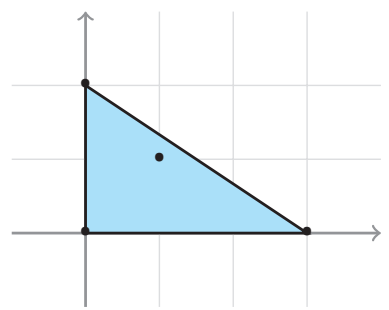

Figure 2. Newton polytope of $f(x, y)=x^{3}+3 x y+y^{2}+1$.

One of the major discoveries of the Moscow Newton polyhedra school is the celebrated BKK theorem named after David Bernstein (younger brother of Joseph Bernstein), Askold Khovanskii, and Anatoli Kushnirenko. Fix a finite set of lattice points $\mathcal{A}=\left\{\alpha_{0}, \ldots, \alpha_{s}\right\} \subset \mathbb{Z}^{n}$ and consider the finite-dimensional vector space $L_{\mathcal{A}}=\left\{f(x)=\sum_{i=0}^{S} c_{i} x^{\alpha_{i}} \mid\right.$ $\left.\forall i, c_{i} \in \mathbb{C}\right\}$ consisting of Laurent polynomials with exponents from $\mathcal{A}$. The convex hull of $\mathcal{A}$, which we denote $\Delta_{\mathcal{A}}$, is the Newton polytope of a generic element of $L_{\mathcal{A}}$.

Theorem 1.2 (BKK). For a generic choice of $f_{1}, \ldots, f_{n} \in L_{\mathcal{A}}$, the number of solutions $x$ in $\left(\mathbb{C}^{*}\right)^{n}$ of the system $f_{1}(x)=\cdots=$ $f_{n}(x)=0$ is the same and is equal to $n ! \operatorname{vol}\left(\Delta_{\mathcal{A}}\right)$, where vol denotes the standard Lebesgue measure in $\mathbb{R}^{n}$.

Remark 1.3. In fact, the above form of this theorem is due to Kushnirenko. Khovanskii has found many different proofs for this theorem (worthy of Guinness Book of Records?). Extending the BKK theorem, he also found formulas in terms of the Newton polytope $\Delta_{\mathcal{A}}$ for genera and Euler characteristics of subvarieties defined by $f_{1}(x)=\cdots=$ $f_{k}(x)=0$, for $k \leq n$, and, as before, generic elements $f_{i} \in L_{\mathcal{A}}$ (see [Hov78]).

Consider the subset $T_{\mathcal{A}} \subset \mathbb{C P}^{s}$ defined by

$$
T_{\mathcal{A}}=\left\{\left(x^{\alpha_{0}}: \cdots: x^{\alpha_{s}}\right) \mid x \in T=\left(\mathbb{C}^{*}\right)^{n}\right\} .
$$

The subset $T_{\mathcal{A}}$ is isomorphic to an algebraic torus. One can show that if the differences of elements in $\mathcal{A}$ generate $\mathbb{Z}^{n}$, then $T_{\mathcal{A}}$ is isomorphic to $T=\left(\mathbb{C}^{*}\right)^{n}$. Let $X_{\mathcal{A}} \subset \mathbb{C P}^{s}$ be the closure of $T_{\mathcal{A}}$. Note that the torus $T=\left(\mathbb{C}^{*}\right)^{n}$ acts on $\mathbb{C P}^{s}$ by

$$
x \cdot\left(z_{0}: \cdots: z_{S}\right)=\left(x^{\alpha_{0}} z_{0}: \cdots: x^{\alpha_{s}} z_{S}\right),
$$

and the variety $X_{\mathcal{A}}$ is the closure of the orbit of $(1: \cdots: 1)$. Recall that the degree of an $n$-dimensional projective variety $X \subset \mathbb{C P}^{S}$ is equal to the number of intersection points of $X$ with a generic plane of codimension $n$. This notion generalizes the degree of a polynomial and provides a measurement for how complex the embedding of $X$ in $\mathbb{C P}^{S}$ is. The BKK theorem can be restated as giving a formula for the degree of $X_{\mathcal{A}} \subset \mathbb{C} \mathbb{P}^{S}$.

Theorem 1.4 (Alternative statement). The degree of $X_{\mathcal{A}} \subset$ $\mathbb{C P}^{S}$ is equal to $n ! \operatorname{vol}\left(\Delta_{\mathcal{A}}\right)$.

Remark 1.5. One of the simplest proofs of the BKK theorem, due to Khovanskii, uses the above restatement and the classical Hilbert theorem on the relation between the degree of a projective variety and the leading term of its Hilbert polynomial. This proof enables a generalization of this theorem to arbitrary systems of equations and is the basis of the theory of Newton-Okounkov bodies (Section $3)$.

Since $X_{\mathcal{A}}$ is the closure of an orbit, it is invariant under the $T$-action. It is a $T$-toric variety in the sense of the following definition.

Definition 1.6. An (abstract) T-toric variety is an irreducible variety with an algebraic $T$-action that has a finite number of $T$-orbits. 
For $X$ a $T$-toric variety there is an open dense orbit $U_{0} \subset$ $X$. After replacing $T$ with $T / T_{0}$, where $T_{0}$ is the $T$-stabilizer of $U_{0}$, without loss of generality we can assume that $U_{0}$ is isomorphic to $T$ itself.

The degree of $X_{\mathcal{A}}$ is not the only geometric information we can obtain from the polytope $\Delta_{\mathcal{A}}$. In general, there is a beautiful correspondence between the algebro-geometry of toric varieties and the combinatorics of convex polytopes. The following is an example of this correspondence.

Theorem 1.7. There is a one-to-one correspondence between the faces of the polytope $\Delta_{\mathcal{A}}$ and the torus orbits in $X_{\mathcal{A}}$.

To make the connection between toric varieties and convex polytopes more tight, one usually assumes $X$ is a normal variety. Some authors include this in the definition of a toric variety (see [Ful93]). The connection between the theory of toric varieties and Newton polytopes was discovered by Khovanskii in now classic papers [Hov77, Hov78].

Remark 1.8. Using the rich correspondence between geometry of toric varieties and combinatorics of convex polytopes, Victor Batyrev famously proved the mirror symmetry conjecture for smooth toric varieties. This is a deep conjecture in algebraic geometry inspired by high-energy physics, and in particular string theory. Batyrev's work is based on the Khovanskii-Danilov computation of Hodge-Deligne numbers using Newton polytopes.

Remark 1.9. The correspondence between toric varieties and convex polytopes has also proven to be an extremely powerful tool in attacking some deep combinatorial problems. Given a sequence $f=\left(f_{0}, \ldots, f_{n-1}\right)$ of nonnegative integers, one asks whether there is an $n$-dimensional polytope $P$ all of whose faces are simplices so that $f_{i}$ is the number of $i$-dimensional faces of $P$ for all $i=0, \ldots, n-$ 1. The Dehn-Sommerville equations give necessary and sufficient conditions on the sequence $f$ for this to hold. Richard Stanley gave a proof of (the necessity of) DehnSommerville equations by interpreting the vector $f$ in terms of the (intersection) cohomology of projective toric varieties (see [Ful93, Section 5.6]). Alternatively Khovanskii found a proof of the Dehn-Sommerville equations based on Morse theory on polytopes/toric varieties.

Interestingly there is a natural (continuous and almost everywhere differentiable) map $\mu_{\mathcal{A}}: X_{\mathcal{A}} \rightarrow \Delta_{\mathcal{A}}$. It is called the moment map of the variety $X_{\mathcal{A}}$. It is a special case of the notion of moment map of a Hamiltonian torus action from symplectic geometry and classical mechanics. Here we regard (the smooth locus of) $X_{\mathcal{A}}$ as a symplectic manifold with respect to the restriction of the standard Fubini-Study form on $\mathbb{C P}^{S}$. This map can be written explicitly (without any knowledge of symplectic geometry required). Given a finite set $\mathcal{A}=\left\{\alpha_{0}, \ldots, \alpha_{s}\right\} \subset \mathbb{Z}^{n}$ the map

$$
\begin{aligned}
\mu_{\mathcal{A}}: & \mathbb{C} \mathbb{P}^{S} \rightarrow \mathbb{R}^{n} \text { is defined by } \\
& \mu_{\mathcal{A}}:\left(z_{0}: \cdots: z_{s}\right) \mapsto \sum_{i=0}^{s}\left(\frac{\left|z_{i}\right|^{2}}{\sum_{j=0}^{S}\left|z_{j}\right|^{2}}\right) \alpha_{i} \in \Delta_{\mathcal{A}} .
\end{aligned}
$$

One easily verifies that $\mu$ is invariant under the action of the topological torus $\left(S^{1}\right)^{n} \subset T=\left(\mathbb{C}^{*}\right)^{n}$. Moreover, $\mu_{\mathcal{A}}\left(\mathbb{C P} \mathbb{P}^{S}\right)=\mu_{\mathcal{A}}\left(X_{\mathcal{A}}\right)=\Delta_{\mathcal{A}}$.

Remark 1.10. The variety $X_{\mathcal{A}}$ inherits a volume form/ measure, called the Liouville measure, from the standard Fubini-Study metric on $\mathbb{C P}^{S}$. One can directly compute the pushforward of the Liouville measure on $X_{\mathcal{A}}$ to $\Delta_{\mathcal{A}}$ and show that the pushforward measure is (a constant multiple of) the Lebesgue measure on $\Delta_{\mathcal{A}}$. From this one can give an elegant proof of the BKK theorem. This proof is due to Khovanskii. For a beautiful account of these ideas and more details, see [Ati83].

Example 1.11 (Baby example). Let $n=1$ and $\mathcal{A}=\{0,1\} \subset$ $\mathbb{Z}$. One sees that $X_{\mathcal{A}}=\mathbb{C P}^{1}$. The moment map $\mu_{\mathcal{A}}$ : $\mathbb{C P}^{1} \rightarrow[0,1]$ is the height function illustrated in Figure 3. The Fubini-Study metric on $\mathbb{C P}^{1}$ is the usual metric on the sphere, and the Liouville measure is just the surface area on the sphere.

Remark 1.12. In the above example, the fact that the pushforward of the surface area on the sphere $\mathbb{C P}^{1}$ (the FubiniStudy form in this case) is equal to the Lebesgue measure was apparently known to Archimedes! It is directly related to Archimedes's theorem on surface area of a cylinder vs. surface area of a sphere. Cicero describes visiting the tomb of Archimedes, on top of which there were a sphere and a cylinder that Archimedes had requested be placed on his tomb to represent his mathematical discoveries.

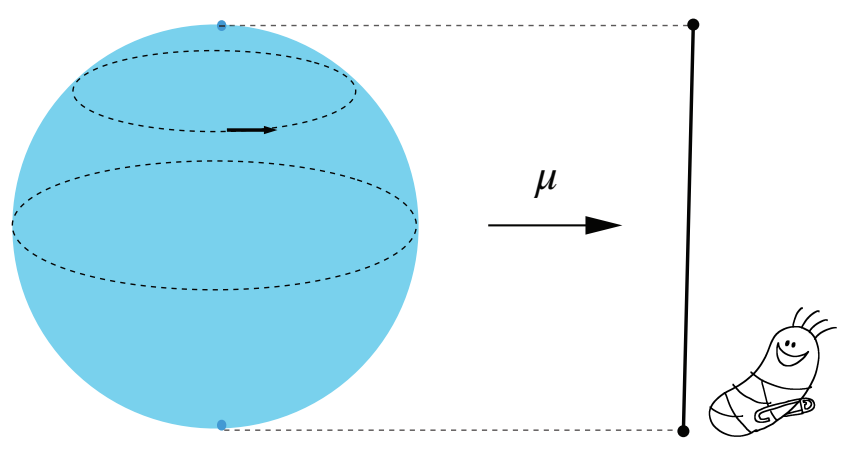

Figure 3. Moment map of the sphere.

\section{The Permutohedron and Associahedron}

Now suppose we have a linear action of a torus $T=\left(\mathbb{C}^{*}\right)^{n}$ on $\mathbb{C P}^{S}$ and a $T$-invariant subvariety $X$ such that the $T$ stabilizer of $X$ is trivial. This yields a moment map $\mu: X \rightarrow$ $\mathbb{R}^{n}$, and its image is a polytope $\Delta_{X}$. (As mentioned before, 


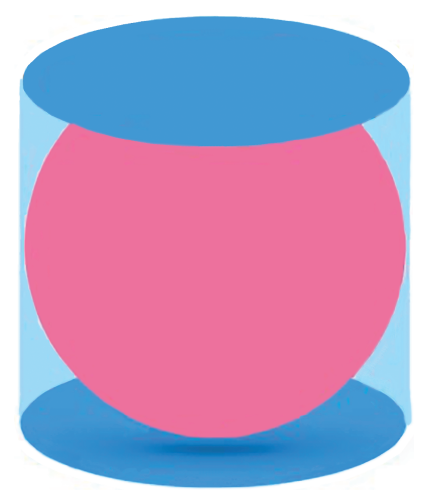

Figure 4. Surface area of the sphere vs. the cylinder.

this is a special case of the moment map from symplectic geometry.) In the previous section we further assumed that $X$ was a toric variety. This implied the existence of an open dense orbit $U_{0} \subset X$ isomorphic to $T$ which in turn forced $\operatorname{dim}(T)=\operatorname{dim}(X)$. In general we may have that $\operatorname{dim}(T)<\operatorname{dim}(X)$, which takes us out of the setting of toric varieties. The polytope $\Delta_{X}$ encodes some geometric information of $X$, but unlike the toric case, the moment map does not yield a tight connection between $\Delta_{X}$ and $X$. Regardless, the moment map construction gives rise to important combinatorics associated to $X$. In this section we give two examples of moment polytopes arising from the study of flag varieties and see they are combinatorially interesting. The first one will be associated to a projective variety that is not toric.

2.1. The permutohedron. The flag variety $F_{n}$ consists of the nested sequences $\{0\}=V_{0} \subsetneq V_{1} \subsetneq \cdots \subsetneq V_{n}=\mathbb{C}^{n}$ of vector subspaces of $\mathbb{C}^{n}$. One can realize this space as a subvariety of a product of projective spaces as follows. An element of $F_{n}$ can be represented by an invertible $n \times n$ matrix $M$ by setting $V_{i}$ to be the row-span of the top $i$ rows of $M$. Given an invertible matrix $M$ and a set $I \subset\{1,2, \ldots, n\}$, let $p_{I}(M)$ be the minor of $M$ given by the top $|I|$ rows and the columns in $I$. For $1 \leq i \leq n$, the homogeneous coordinates $\left(p_{I}(M)|| I \mid=i\right) \in \mathbb{C P}^{(}\left(\begin{array}{l}n \\ i\end{array}\right)-1$ are called the Plücker coordinates of the subspace $V_{i}$. Sending $V_{1}, \ldots, V_{n-1}$ to their Plücker coordinates yields an embedding

$$
F_{n} \hookrightarrow \mathbb{C P}^{\left(\begin{array}{l}
n \\
1
\end{array}\right)-1} \times \cdots \times \mathbb{C P}^{\left(\begin{array}{c}
n \\
n-1
\end{array}\right)-1},
$$

and the image is a closed subvariety. To realize $F_{n}$ as a projective variety in $\mathbb{C P}\left(\begin{array}{c}n \\ 1\end{array}\right) \times \cdots \times\left(\begin{array}{c}n \\ n-1\end{array}\right)-1$, we can apply the Segre embedding multiple times.

Define a torus action on the Plücker coordinates as follows. Realize the torus $\left(\mathbb{C}^{*}\right)^{n}$ as the group $\mathcal{D}$ of invertible diagonal $n \times n$ matrices. Given $D \in \mathcal{D}$ and an invertible matrix $M$, the action of $D$ on the Plücker coordinates $p_{I}(M)$ sends them to the Plücker coordinates of $M D$. This action comes from a linear action of $\mathcal{D}$ on $\mathbb{C P}\left(\begin{array}{l}n \\ 1\end{array}\right) \times \cdots \times\left(\begin{array}{l}n \\ n\end{array}\right)-1$.
Furthermore, $F_{n}$ is a $\mathcal{D}$-invariant subvariety and therefore has a moment map. The image of this map is the permutohedron $\mathrm{P}_{n}$.

The permutohedron $\mathrm{P}_{n}$ is the convex hull of the $n$ ! permutations of $(0,1, \ldots, n-1)$. Actually all of these points are vertices of $\mathrm{P}_{n}$. This polytope is the Newton polytope of the Vandermonde determinant

$$
\operatorname{det}\left[\begin{array}{cccc}
1 & 1 & \cdots & 1 \\
x_{1} & x_{2} & \cdots & x_{n} \\
\vdots & \vdots & & \vdots \\
x_{1}^{n-1} & x_{2}^{n-1} & \cdots & x_{n}^{n-1}
\end{array}\right]=\prod_{1 \leq i<j \leq n}\left(x_{j}-x_{i}\right) .
$$

The combinatorics of $\mathrm{P}_{n}$ reflects some of the geometry of the flag variety. For example, the vertices and edge directions of $\mathrm{P}_{n}$ encode the $T$-equivariant cohomology (and hence the usual cohomology) of $F_{n}$.

Remark 2.1. More generally, there is a large class of varieties with a torus action for which one can give an elegant description of their cohomology rings in terms of the combinatorial data of vertices and edges of their moment graphs. This is the class of GKM varieties named after Mark Goresky, Robert Kottwitz, and Robert MacPherson. A $T$-variety $X$ is a GKM variety if it has a finite number of $T$-fixed points, a finite number of $T$-invariant curves, and the $T$-action is socalled equivariantly formal. Examples include flag varieties and toric varieties.

There is a classical formula for the volume of the permutohedron. Let $H_{n}$ be the affine hyperplane given by $x_{1}+\cdots+x_{n}=\frac{n(n-1)}{2}$; note that $\mathrm{P}_{n}$ lies on the affine hyperplane $H_{n}$. The volume of $\mathrm{P}_{n}$ as a polytope in $H_{n}$ (normalized so that every primitive parallelepiped in $H_{n} \cap \mathbb{Z}^{n}$ has volume 1 ) equals $n^{n-2}$. This is equal to the number of trees on $n$ labeled vertices. However, since $F_{n}$ is not a toric variety, the BKK theorem does not apply and $n^{n-2}$ is not the degree of $F_{n}$. The volume of $\mathrm{P}_{n}$ equals the degree of the largest $T$-toric subvariety in $F_{n}$.
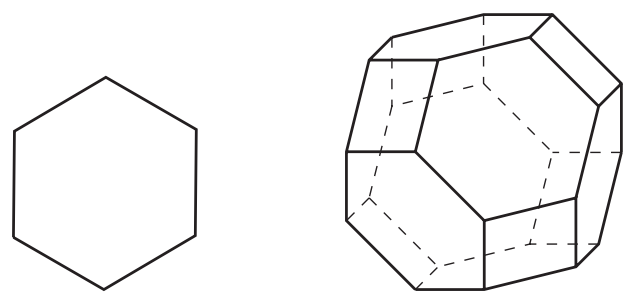

Figure 5. From left to right, the permutohedra $P_{3}$ and $P_{4}$.

2.2. The associahedron. Consider the ways to put parentheses on $t_{1} \cdots t_{n}$ so that we end up with binary products. These products can be arranged into a polyhedral complex, called the associahedron $\mathrm{A}_{n}$. Any balanced placement of parentheses yields a face with vertices the binary products that contain the given parentheses. For example, 


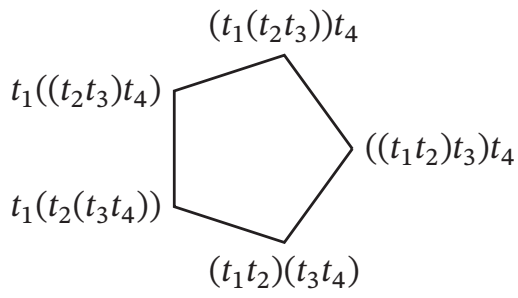

Figure 6. A realization of $A_{4}$.

$t_{1}\left(t_{2} t_{3} t_{4}\right)$ corresponds to the edge with vertices $t_{1}\left(t_{2}\left(t_{3} t_{4}\right)\right)$ and $t_{1}\left(\left(t_{2} t_{3}\right) t_{4}\right)$. See Figure 6 for an example. An alternative description of the associahedron $A_{n}$ is given by considering the vertices to be triangulations of an $(n+1)$-gon where two triangulations are adjacent if you can obtain one by flipping a diagonal of the other; see Figure 7 for an example.

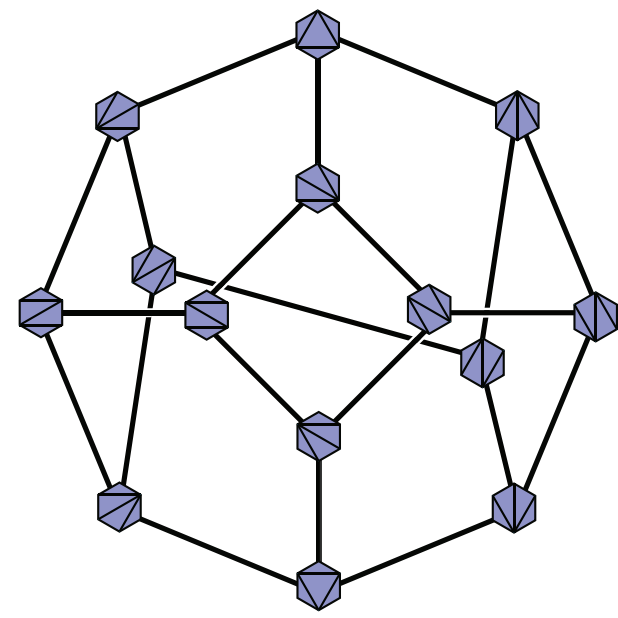

Figure 7. The polyhedral complex with vertices triangulations of a regular hexagon.

A realization of $A_{n}$ is an $(n-2)$-dimensional polytope whose face structure equals the face structure of $A_{n}$. There are many different polytopes that can arise in this way; the paper [CSZ15] has a survey about the different realizations. The Newton polytope of $\prod_{1 \leq i<j \leq n-1}\left(x_{i}+x_{i+1}+\cdots+x_{j}\right)$ is Jean-Louis Loday's realization of $A_{n}$. The toric variety of Loday's realization of $A_{n}$ can be constructed using concepts from the flag variety; see [Esc16]. For brevity we describe only the case $n=4$. Let $e_{1}, e_{2}, e_{3}$ be the standard basis vectors of $\mathbb{C}^{3}$. Consider the variety $B$ consisting of the tuples $\left(V_{1,1}, V_{2,1}, V_{2,2}\right)$ of vector spaces such that the following incidences hold:

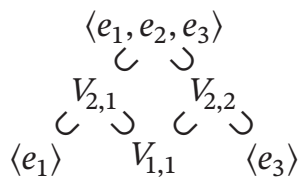

One can then use the Plücker embedding and multiple Segre embeddings to embed $B$ into $\mathbb{P}\left(\begin{array}{l}3 \\ 1\end{array}\right) \times\left(\begin{array}{l}3 \\ 2\end{array}\right) \times\left(\begin{array}{l}3 \\ 2\end{array}\right)-1$.
Schubert varieties are subvarieties of the $F_{n}$ defined by imposing conditions on how the flags intersect the coordinate subspaces of $\mathbb{C}^{n}$. Varieties generalizing the one above can be used to resolve singularities of transverse intersections of Schubert varieties.

Returning to the case at hand, the action of $\mathcal{D}=\left(\mathbb{C}^{*}\right)^{3}$ on $F_{3}$ induces an action of $\mathcal{D}$ on $B$ and a moment map. The moment polytope of the image is Loday's realization of $A_{4}$. It turns out that the dimension of $B$ is equal to the dimension of $A_{4}$, which implies that this variety is actually the toric variety of Loday's realization of $A_{4}$.

\section{Newton-Okounkov Bodies}

The success of toric methods encouraged algebraic geometers to try to extend the scope of convex geometric methods in algebraic geometry. Many of the results about toric varieties have been extended to varieties with actions of so-called reductive groups. These are the complex algebraic counterparts of compact Lie groups and include familiar examples from linear algebra such as $\operatorname{GL}(n, \mathbb{C})$ and $\operatorname{SL}(n, \mathbb{C})$. A large class of varieties with reductive group actions that extends that of toric varieties is the class of spherical varieties. (We caution that the adjective spherical here is not directly related to sphere, but rather to spherical functions from representation theory.) The BKK theorem has been generalized to spherical varieties by Michel Brion and Boris Kazarnovskii and to more general reductive group actions by Kaveh and Khovanskii (see [KK12b] and references therein).

Far more generally, the theory of Newton-Okounkov bodies extends the BKK theorem to arbitrary projective varieties. Let $X \subset \mathbb{C P}^{s}$ be an $n$-dimensional projective variety. Generalizing the BKK formula for degree of $X_{\mathcal{A}}$, we would like to construct a convex body (i.e., a convex compact subset) $\Delta \subset \mathbb{R}^{n}$ such that its volume gives the degree of $X$. In this full generality $\Delta$ may not be a polytope but only a convex body. To do this we need an extra choice of a function $v: \mathbb{C}(X) \backslash\{0\} \rightarrow \mathbb{Z}^{n}$ satisfying the properties of a valuation on the field of rational functions $\mathbb{C}(X)$. Instead of giving the abstract definition of a valuation (from commutative algebra), we explain the geometric construction of a typical valuation on the field of rational functions. It is a generalization of the familiar notion of leading term of a polynomial.

First we equip the additive group $\mathbb{Z}^{n}$ with the lexicographic order. Pick a smooth point $p$ in $X$ and let $\left(u_{1}, \ldots, u_{n}\right)$ be a system of parameters at $p$. That is, the $u_{i}$ are rational functions that are regular at $p$ such that $u_{1}(p)=\cdots=u_{n}(p)=0$, and their differentials at $p$ are linearly independent (in other words, they generate the maximal ideal of $p$ ). It is well known that every rational function $f \in \mathbb{C}(X)$ can be uniquely expressed as a formal Laurent series in the $u_{i}$, that is, $f=\sum_{\alpha=\left(a_{1}, \ldots, a_{n}\right)} c_{\alpha} u_{1}^{a_{1}} \cdots u_{n}^{a_{n}}$. 
Then we define $v: \mathbb{C}(X) \backslash\{0\} \rightarrow \mathbb{Z}^{n}$ by

$$
v(f)=\min \left\{\alpha \mid c_{\alpha} \neq 0\right\} .
$$

Here the minimum is taken with respect to the lexicographic order on $\mathbb{Z}^{n}$ (one shows that the minimum always exists in this case). Note that the choice of $v$ is independent of the choice of embedding of $X$ into projective space.

Let $\mathbb{C}[X]$ be the homogeneous coordinate ring of $X$. If $I \subset \mathbb{C}\left[x_{0}, \ldots, x_{s}\right]$ is the homogeneous ideal defining $X \subset$ $\mathbb{C P}^{S}$, then $\mathbb{C}[X]=\mathbb{C}\left[x_{0}, \ldots, x_{s}\right] / I$ and it is a $\mathbb{Z}_{\geq 0}$-graded algebra. Denote by $\mathbb{C}[X]_{m}$ the $m$ th graded piece of $\mathbb{C}[X]$ so that

$$
\mathbb{C}[X]=\bigoplus_{m \in \mathbb{Z}_{\geq 0}} \mathbb{C}[X]_{m} .
$$

Using the valuation $v$ we would like to associate a convex body $\Delta$ to the graded algebra $\mathbb{C}[X]$. Fix a nonzero element $h \in \mathbb{C}[X]_{1}$. First, we construct a semigroup $S=S(X, v) \subset$ $\mathbb{Z}_{>0} \times \mathbb{Z}^{n}$ by

$$
S=\bigcup_{m>0}\left\{\left(m, v\left(f / h^{m}\right)\right) \mid 0 \neq f \in \mathbb{C}[X]_{m}\right\} .
$$

Let $C \subset \mathbb{R}^{n+1}$ be the closure of the convex hull of $S \cup\{0\}$.

Definition 3.1. The Newton-Okounkov body $\Delta=\Delta(X, v)$ of $X \subset \mathbb{C P}^{S}$ is the intersection of the convex cone $C$ with the hyperplane $x_{1}=1$ in $\mathbb{R}^{n+1}$. One shows that $\Delta$ is bounded and hence a convex body.

The construction of $\Delta(X, v)$ appears (in passing) in [Oko96, Oko03]. It was defined in a more general setting and systematically studied in [LM09, KK12a].

The main theorem regarding Newton-Okounkov bodies is a far generalization of the BKK theorem (see [Oko03, LM09, KK12a]).

Theorem 3.2 (Okounkov, Lazarsfeld-Mustaţă, KavehKhovanskii). With notation as above,

$$
\operatorname{deg}(X)=n ! \operatorname{vol}(\Delta) .
$$

An important application of realizing degree as volume of a convex body is that one can apply the celebrated Brunn-Minkowski inequality (which is an inequality about volumes of subsets in Euclidean space) to Newton-Okounkov bodies to obtain a simple proof of a deep fact, known as the Hodge inequality, about intersection numbers of hypersurfaces on varieties (see [Oko03, LM09, KK12a]).

Example 3.3. Let $X \subset \mathbb{C P}^{2}$ be a plane algebraic curve defined by a homogeneous polynomial of degree $d$. Let $v$ be the order of vanishing at a smooth point in $X$. One shows, by direct computation or using Theorem 3.2, that $\Delta(X, v)$ is the line segment $[0, d]$.
Example 3.4. With notation as before, let $X \subset \mathbb{C P}^{s}$ be a projective variety with degree $d$. Let $v$ be the valuation obtained from a system of parameters corresponding to $n=\operatorname{dim}(X)$ hyperplane sections in general position. Then $\Delta(X, v)$ is the simplex in $\mathbb{R}^{n}$ with vertices $0, e_{1}, \ldots, e_{n-1}$, and $d e_{n}$, where $\left\{e_{1}, \ldots, e_{n}\right\}$ is the standard basis.

It is possible that $\Delta(X, v)$ is not a polytope (see [LM09, Section 6.3]). For random choices of $X$ and $v$ one expects that the convex body $\Delta(X, v)$ is not a polytope, $\mathrm{cf}$. work of Küronya-Lozovanu-Maclean.

Remark 3.5. One can also define local versions of NewtonOkounkov bodies. In commutative algebra terms, to a primary ideal $I$ in a local algebra $R$ one can associate a convex set $\Gamma(I)$ inscribed in a cone $C(R)$ such that the volume of its complement gives the Samuel multiplicity e(I) (see [KK14] as well as work of Dale Cutkosky).

The notion of a toric degeneration provides a geometric explanation for why Theorem 3.2 holds.

Definition 3.6. A toric degeneration of an embedded projective variety $X \subset \mathbb{C P}^{s}$ is a family $\pi: \mathfrak{X} \rightarrow \mathbb{C}$ where $\mathfrak{X} \subset \mathbb{C P}^{s} \times \mathbb{C}$ and $\pi$ is the projection on the second factor, such that the following hold:

(1) The family is trivial over $\mathbb{C}^{*}$ with fiber isomorphic to $X$. That is, $\pi^{-1}\left(\mathbb{C}^{*}\right) \cong X \times \mathbb{C}^{*}$.

(2) The fibers $X_{t}:=\pi^{-1}(t), t \in \mathbb{C}$, are all reduced and irreducible (by (1) it suffices that $X_{0}$ is reduced and irreducible).

(3) The fiber $X_{0}=\pi^{-1}(0)$ (special fiber) is a toric variety with respect to an action of $T=\left(\mathbb{C}^{*}\right)^{n}$ induced from a linear action of $T$ on $\mathbb{C P}^{S}$.

A toric degeneration is a "deformation" of a given variety to a toric variety such that some useful intersection theoretic data, in particular the degree, are preserved under the deformation, hence enabling us to obtain some geometric information about the embedding $X \subset \mathbb{C P}^{s}$ from its degenerated toric variety.

We should point out that degenerations of curves is a classical and very well-studied subject.

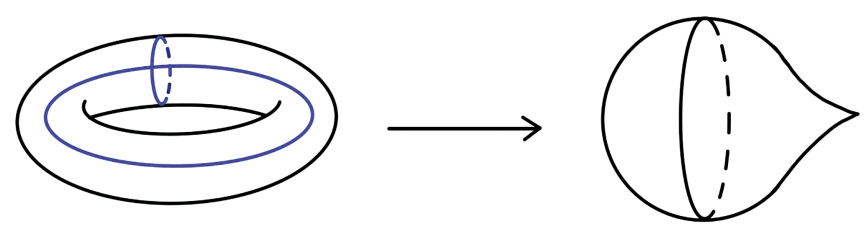

Figure 8. The family $\left\{((x: y: z), t) \mid y^{2} z=x^{3}+t^{3} z^{3}\right\} \subset \mathbb{C P} \mathbb{P}^{2} \times \mathbb{C}$ gives a toric degeneration of the elliptic curve $y^{2} z=x^{3}+z^{3}$ to the singular toric variety $y^{2} z=x^{3}$. Topologically, a donut shape degenerates to a pinched sphere. The two circles around the arm and the hole of the donut contract to a point on the pinched sphere. 
Using standard methods from commutative algebra (namely, Rees algebra associated to a valuation) it can be shown that whenever the semigroup $S=S(X, v)$ is finitely generated $X \subset \mathbb{C P}^{s}$ admits a toric degeneration to the toric variety $X_{\mathcal{A}} \subset \mathbb{C P}^{S}$ associated to a finite set $\mathcal{A}$ of generators of $S$ [AND13]. To be precise we want $S$ to be generated in level 1.

Remark 3.7. These toric degenerations can be used in symplectic geometry for constructions of full-dimensional Hamiltonian torus actions as well as to obtain general results and constructions about symplectic ball embeddings from symplectic topology [HK15, Kav19].

Remark 3.8. The Newton-Okounkov bodies of the flag manifold have been computed by various mathematicians with respect to some geometric valuations. For example, in [Kav15] the author gives valuations for which the resulting Newton-Okounkov bodies are the string polytopes of Peter Littelmann, Arkady Berenstein, and Andrei Zelevinsky, a generalization of the GelfandZetlin polytopes. Gelfand-Zetlin polytopes are in oneto-one correspondence with irreducible representations of $\operatorname{GL}(n, \mathbb{C})$. Moreover, the number of integral points in a given Gelfand-Zetlin polytope is equal to the dimension of the irreducible representation it corresponds to. Valentina Kiritchenko gave alternative valuations such that the resulting Newton-Okounkov bodies are FeiginFourier-Littelmann-Vinberg polytopes. Also, polytopes of Nakashima-Zelevinsky were realized as NewtonOkounkov bodies of flag varieties by Naoki Fujita and Hironori Oya. There are also recent interesting connections between Newton-Okounkov bodies and cluster algebras (see [RW19] as well as [KM19, p. 298] and references therein).

Given an embedded projective variety $X \subset \mathbb{C P}^{s}$, one is interested to know when there is a valuation $v$ on $\mathbb{C}(X)$ such that the corresponding value semigroup $S$ is finitely generated. In [KM19] the authors provide a criterion for this in terms of tropical geometry and Gröbner theory.

With notation as before let $I \subset \mathbb{C}\left[x_{0}, \ldots, x_{s}\right]$ be the homogeneous ideal defining $X \subset \mathbb{C P}^{S}$. Let us recall the basic notion of initial form of a polynomial from the Gröbner basis theory. Take $w \in \mathbb{Q}^{s+1}$ and let $f(x)=\sum_{\alpha \in \mathbb{Z}_{\geq 0}^{s+1}} c_{\alpha} x^{\alpha}$ be a polynomial. The initial form $\operatorname{in}_{w}(f)$ is defined to be the sum of terms $c_{\alpha} x^{\alpha}$ where the dot product $\langle w, \alpha\rangle$ is minimum. More precisely, let $m_{0}=\min \left\{\langle w, \alpha\rangle \mid c_{\alpha} \neq 0\right\}$. Then

$$
\operatorname{in}_{w}(f)=\sum_{\langle w, \alpha\rangle=m_{0}} c_{\alpha} x^{\alpha} .
$$

The initial ideal of $I$ with respect to the weight $w$ is the ideal generated by the $\operatorname{in}_{w}(f)$ for all $0 \neq f \in I$.

Given a homogeneous ideal $I$, let us say that two vectors $w_{1}, w_{2} \in \mathbb{Q}^{S+1}$ are equivalent if $\operatorname{in}_{w_{1}}(I)=\operatorname{in}_{w_{2}}(I)$. It is

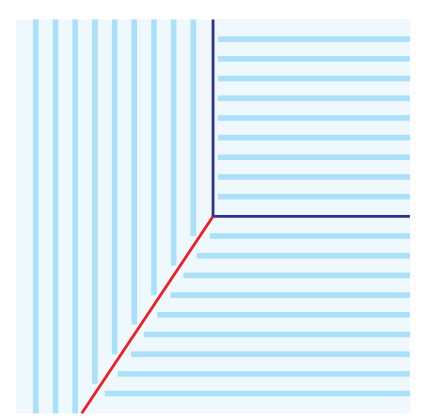

Figure 9. The Gröbner fan of the ideal $\left(y^{2} z-x^{3}+7 x z^{2}-2 z^{3}\right)$. The ray colored red is the only (full-dimensional) prime cone.

a well-known result that the equivalence classes partition $\mathbb{Q}^{s+1}$ into relatively open rational polyhedral cones. This partition is usually referred to as the Gröbner fan of $I$, and we denote it by $\Sigma(I)$.

We will say that a cone $C \in \Sigma(I)$ is a prime cone if the corresponding initial ideal $\operatorname{in}_{C}(I)=\operatorname{in}_{w}(I), \forall w \in C$, is a prime ideal in $\mathbb{C}\left[x_{0}, \ldots, x_{s}\right]$. One of the main results in [KM19] establishes a correspondence between (full rank) valuations on $\mathbb{C}[X]$ whose corresponding value semigroup is finitely generated and (full-dimensional) prime cones in the Gröbner fan of $I$. Thus to each such cone one can naturally associate a Newton-Okounkov body (which in this case is in fact a polytope) $\Delta_{C}$. The following example is from [KM19, p. 300].

Example 3.9. Consider the ideal $I=\left(y^{2} z-x^{3}+7 x z^{2}-2 z^{3}\right)$ defining an elliptic curve $E \subset \mathbb{C P}^{2}$. The Gröbner fan of $I$ lives in $\mathbb{R}^{3}$. Since $I$ is a homogeneous ideal, one sees that every cone in the Gröbner fan is invariant under adding scalar multiples of the vector $(1,1,1)$. Thus we can think of the Gröbner fan as living in $\mathbb{R}^{3} /\langle(1,1,1)\rangle \cong \mathbb{R}^{2}$. It consists of the seven cones (three 2-dimensional cones, three rays, and the origin) in Figure 9. One computes that only the ray colored red is a (full-dimensional) prime cone.

The above correspondence leads to the following question: How does the Newton-Okounkov polytope change if we cross from one prime cone to an adjacent prime cone in the Gröbner fan? The preprint [EH19] gives the following answer:

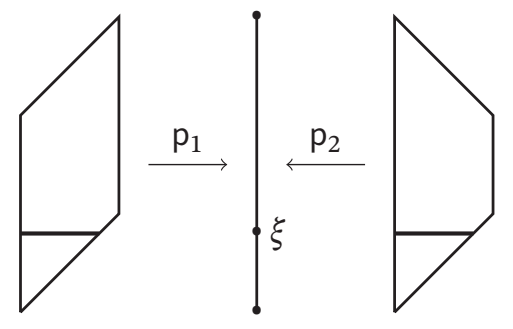

Figure 10. Two Newton-Okounkov polytopes that project onto the same polytope and such that the fibers under the projection maps are the same length. 
Theorem 3.10 (Wall-crossing, Escobar-Harada). Suppose that $C_{1}, C_{2}$ are two prime cones in $\Sigma(I)$ that share a codimension-1 face. There exist a polytope $\Delta$ of $\operatorname{dim}(\Delta)+1=$ $\operatorname{dim}\left(\Delta_{C_{1}}\right)=\operatorname{dim}\left(\Delta_{C_{2}}\right)$ and two natural surjective projections

$$
\Delta_{C_{1}} \stackrel{\mathrm{p}_{1}}{\longrightarrow} \Delta \stackrel{\mathrm{p}_{2}}{\longleftarrow} \Delta_{C_{2}}
$$

such that the fibers of $\mathrm{p}_{1}$ and $\mathrm{p}_{2}$ of any point $\xi \in \Delta$ are 1dimensional polytopes of the same Euclidean length (up to a global constant). Moreover, there exists a piecewise linear bijection $\mathrm{F}: \Delta_{C_{1}} \rightarrow \Delta_{C_{2}}$ which makes the following diagram commute:

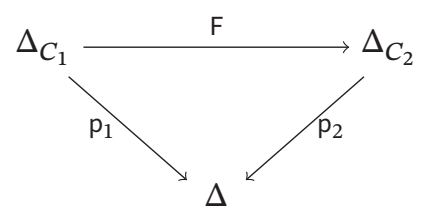

It was observed by Nathan Ilten and Christopher Manon in 2017 that the geometric wall-crossing phenomenon for Newton-Okounkov bodies, as described above, can also be derived from the theory of complexity-one Tvarieties.

\section{References}

[AND13] Dave Anderson, Okounkov bodies and toric degenerations, Math. Ann. 356 (2013), no. 3, 1183-1202. MR3063911

[Ati83] M. F. Atiyah, Angular momentum, convex polyhedra and algebraic geometry, Proc. Edinburgh Math. Soc. (2) 26 (1983), no. 2, 121-133. MR705256

[CSZ15] Cesar Ceballos, Francisco Santos, and Günter M. Ziegler, Many nonequivalent realizations of the associahedron, Combinatorica 35 (2015), no. 5, 513-551. MR3437894

[Esc16] Laura Escobar, Brick manifolds and toric varieties of brick polytopes, Electron. J. Combin. 23 (2016), no. 2, Paper 2.25, 18. MR3512647

[EH19] Laura Escobar and Megumi Harada, Wall-crossing for Newton-Okounkov bodies and the tropical Grassmannian, arXiv:1912.04809, 2019.

[Ful93] William Fulton, Introduction to toric varieties, Annals of Mathematics Studies, vol. 131, Princeton University Press, Princeton, NJ, 1993. The William H. Roever Lectures in Geometry. MR1234037

[HK15] Megumi Harada and Kiumars Kaveh, Integrable systems, toric degenerations and Okounkov bodies, Invent. Math. 202 (2015), no. 3, 927-985. MR3425384

[Hov77] A. G. Hovanskiǔ, Newton polyhedra, and toroidal varieties, Funkcional. Anal. i Priložen. 11 (1977), no. 4, 56-64, 96. MR0476733

[Hov78] A. G. Hovanskiü, Newton polyhedra, and the genus of complete intersections, Funktsional. Anal. i Prilozhen. 12 (1978), no. 1, 51-61. MR487230

[Kav15] Kiumars Kaveh, Crystal bases and Newton-Okounkov bodies, Duke Math. J. 164 (2015), no. 13, 2461-2506. MR3405591
[Kav19] Kiumars Kaveh, Toric degenerations and symplectic geometry of smooth projective varieties, J. Lond. Math. Soc. (2) 99 (2019), no. 2, 377-402. MR3939260

[KK12a] Kiumars Kaveh and A. G. Khovanskii, NewtonOkounkov bodies, semigroups of integral points, graded algebras and intersection theory, Ann. of Math. (2) 176 (2012), no. 2, 925-978. MR2950767

[KK12b] Kiumars Kaveh and Askold G. Khovanskii, Convex bodies associated to actions of reductive groups, Mosc. Math. J. 12 (2012), no. 2, 369-396, 461. MR2978761

[KK14] Kiumars Kaveh and Askold Khovanskii, Convex bodies and multiplicities of ideals, Proc. Steklov Inst. Math. 286 (2014), no. 1, 268-284. Reprint of Tr. Mat. Inst. Steklova 286 (2014), 291-307. MR3482603

[KM19] Kiumars Kaveh and Christopher Manon, Khovanskii bases, higher rank valuations, and tropical geometry, SIAM J. Appl. Algebra Geom. 3 (2019), no. 2, 292-336. MR3949692

[LM09] Robert Lazarsfeld and Mircea Mustaţă, Convex bodies associated to linear series, Ann. Sci. Éc. Norm. Supér. (4) 42 (2009), no. 5, 783-835. MR2571958

[MS15] Diane Maclagan and Bernd Sturmfels, Introduction to tropical geometry, Graduate Studies in Mathematics, vol. 161, American Mathematical Society, Providence, RI, 2015. MR3287221

[Oko03] Andrei Okounkov, Why would multiplicities be logconcave?, The orbit method in geometry and physics (Marseille, 2000), 2003, pp. 329-347. MR1995384

[Oko96] Andrei Okounkov, Brunn-Minkowski inequality for multiplicities, Invent. Math. 125 (1996), no. 3, 405-411. MR1400312

[RW19] K. Rietsch and L. Williams, Newton-Okounkov bodies, cluster duality, and mirror symmetry for Grassmannians, Duke Math. J. 168 (2019), no. 18, 3437-3527. MR4034891

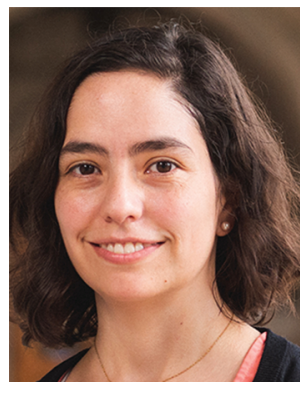

Laura Escobar

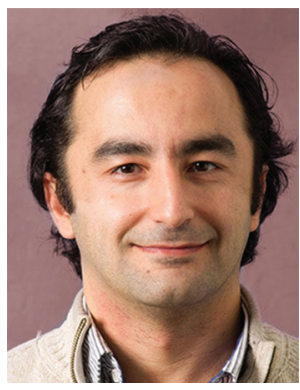

Kiumars Kaveh

\section{Credits}

Figure 1 was taken from https://cud1.7ib.cam.ac.uk /view/MS-ADD-03977/18, Reproduced by kind permission of the Syndics of Cambridge University Library.

All other figures were created by the authors.

Photo of Laura Escobar is by Sean Garcia and is courtesy of Washington University in St. Louis.

Photo of Kiumars Kaveh is courtesy of the University of Pittsburgh. 